\title{
A Case of Fluorine-18 Fluorodeoxyglucose Positron Emission Tomography/ Computed Tomography Imaging of Eosinophilic Granulomatosis With Polyangiitis Combined With Non-tuberculous Mycobacterium
}

\author{
Juhyung Kim${ }^{1}$, Man-Hoon Han², Sang Jin Lee ${ }^{1}$ \\ ${ }^{1}$ Department of Internal Medicine, School of Medicine, Kyungpook National University, Daegu, Korea \\ ${ }^{2}$ Department of Pathology, School of Medicine, Kyungpook National University, Daegu, Korea
}

\section{Doi: 10.12890/2019_001299 - European Journal of Case Reports in Internal Medicine - @ EFIM 2019}

Received: 28/09/2019

Accepted: $15 / 10 / 2019$

Published: 22/11/2019

How to cite this article: Kim J, Han MN, Lee SG. A case of fluorine-18 fluorodeoxyglucose positron emission tomography/computed tomography imaging of eosinophilic granulomatosis with polyangiits combined with non-tuberculous mycobacterium. EJCRIM 2019;6: doi:10.12890/2019_001299.

Conflicts of Interests: The Authors declare that there are no competing interest

This article is licensed under a Commons Attribution Non-Commercial 4.0 License

\section{ABSTRACT}

Fluorine-18 fluorodeoxyglucose positron emission tomography/computed tomography (18FDG-PET/CT) has been used to diagnose vasculitis, tuberculosis and malignancy. As PET/CT scans show hotspots during an activation of clinically suspected lesions, they are widely used for diagnosis. However, there are rare cases of PET/CT images for vasculitis combined with tuberculosis. Here, we report a case of eosinophilic granulomatosis with polyangiitis in a patient with disseminated non-tuberculous mycobacterial infection in multiple sites mimicking metastatic malignancy and describe the associated PET/CT scan findings before and after treatment.

\section{LEARNING POINTS}

- $\quad \mathrm{PET} / \mathrm{CT}$ can better inform accurate diagnosis for detecting biopsy sites and differentiating diseases.

- Eosinophilic granulomatosis with polyangiitis and non-tuberculous mycobacterial infection have been diagnosed simultaneously in this case.

- The therapeutic response can be assessed with PET/CT in patients with eosinophilic granulomatosis with polyangiitis.

\section{KEYWORDS}

Positron emission tomography/computed tomography, non-tuberculous mycobacterium, eosinophilic granulomatosis with polyangiitis

\section{INTRODUCTION}

Fluorine-18 fluorodeoxyglucose positron emission tomography/computed tomography (18FDG-PET/CT) may be used for diagnosis and follow-up of autoimmune diseases, infection and malignanc ${ }^{[1-7]}$. As PET/CT scans show hotspots during an activation of clinically suspected disease activity, they are helpful for detecting biopsy sites and differentiating diseases ${ }^{[8]}$.

Prior clinical work has suggested that infectious diseases may be linked to the development of vasculitis ${ }^{[9-11]}$. However, little is known about the mechanisms by which infectious pathogens trigger vasculitis. We present an eosinophilic granulomatosis with polyangiitis (EGPA) patient who had disseminated non-tuberculous mycobacteria (NTM) infection and describe the associated PET/CT scan features. To the best of the authors' knowledge, this is the first reported case of a patient with EGPA combined with disseminated NTM infection with findings in multiple sites mimicking metastatic malignancy 


\section{CASE DESCRIPTION}

A 69-year-old male patient presented with complaints of purulent nasal discharge and hard palate perforation (Fig. 1A) 6 months prior to admission to our hospital.

He was subsequently diagnosed with sinusitis and underwent endoscopic sinus surgery. However, the nasal discharge persisted. After admission to our facility, his examination revealed a temperature of $37.8^{\circ} \mathrm{C}$, blood pressure of $124 / 76 \mathrm{mmHg}$, a pulse rate of $83 \mathrm{beats} /$ minute, and purulent skin ulcers in the right hand and left knee (Fig. 1B).

Neurologic examination showed intact strength and normal sensation, and the lung sounds were clear without wheezing. The anti-neutrophil cytoplasmic antibody (ANCA) test was negative and the anti-nuclear antibody test was positive (1:320). However, anti-double-stranded deoxyribonucleic acid (dsDNA) antibody, complement C3/C4, immunoglobulin G4 and creatinine were all within the normal range. The white blood cell count, C-reactive protein levels and the erythrocyte sedimentation rate were elevated as follows: $21,456 / \mathrm{mm}^{3}$ (reference

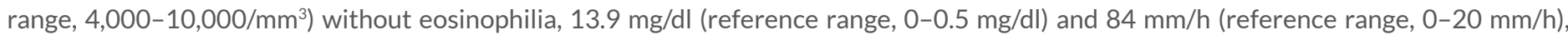
respectively. There was no haematuria or proteinuria present on urinalysis.

Nasal endoscopy revealed a bloody crust with severe inflammation (Fig. 1C).
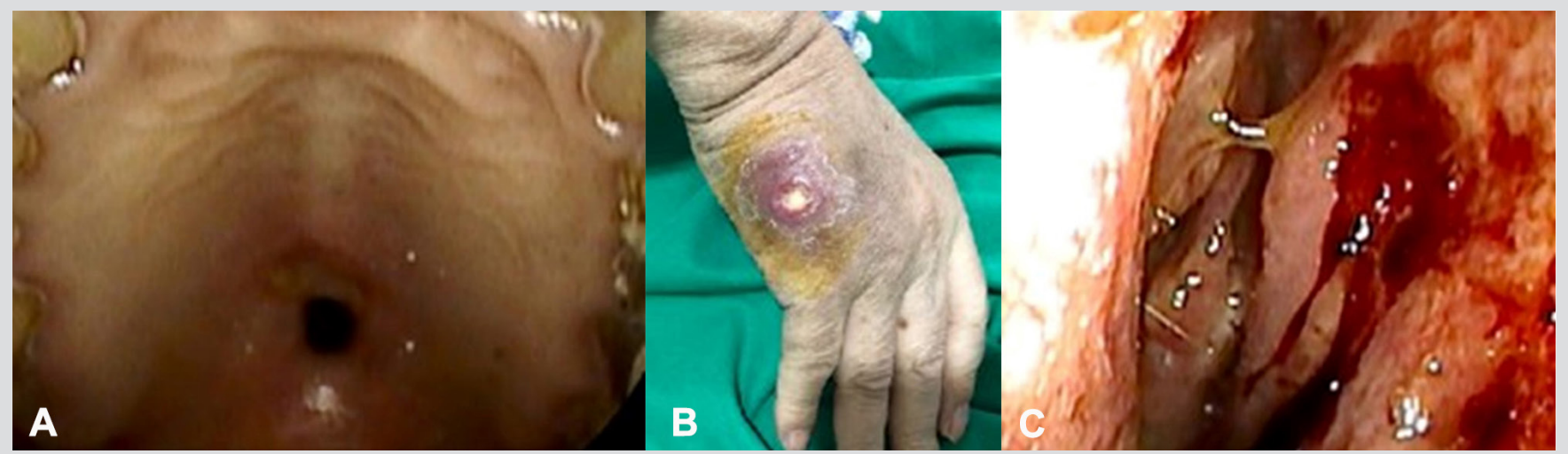

Figure 1. Appearance of the patient at admission. Hard palate perforation (A) and a skin ulcer on the right hand (B) were observed. Nasal endoscopy demonstrated a bloody and purulent discharge with severe inflammation (C)

A bone marrow biopsy was carried out to exclude malignancy. There was no evidence of a malignant tumour and acid-fast bacilli staining was negative. However, biopsies of the right hand and left knee abscesses and of the nasal cavity showed acute gangrenous inflammation, and the NTM, M. intracellulare, were grown in the culture of these specimens. We performed second biopsies on the nasal cavity and left iliac bone in regions that demonstrated high uptake on the PET/CT scan. Granulomatous inflammation with multinucleated giant cells and vasculitis were seen in the nasal cavity bone (Fig. 3A,B). Severe inflammation with multiple eosinophils was seen in the left iliac bone (Fig. 3C).

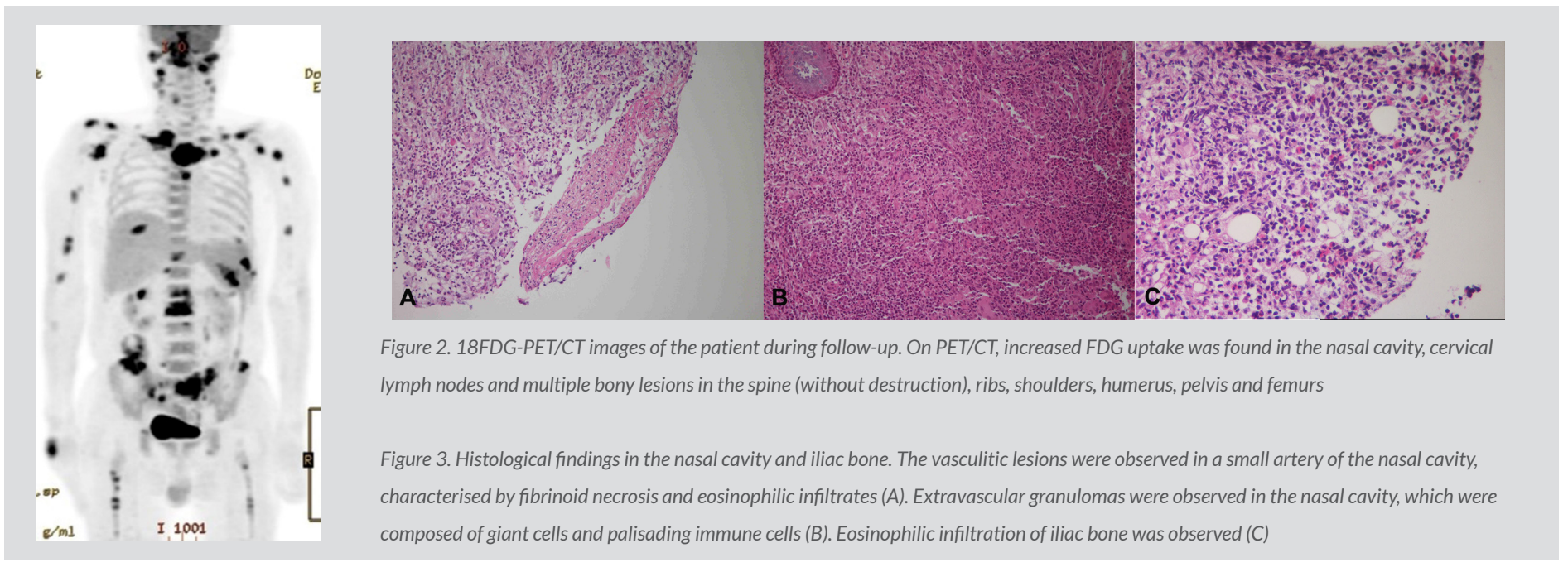


During his hospital stay, the patient complained of a right foot drop without any abnormality of sensation. A needle EMG and nerve conduction study showed a right common peroneal neuropathy. The patient was diagnosed with EGPA combined with NTM infection. Three months after treatment with cyclophosphamide, a steroid and anti-NTM medication (clarithromycin, ethambutol and rifampin), the abscesses in the right hand and left knee resolved with improvement noted on follow-up PET/CT (Fig. 4).

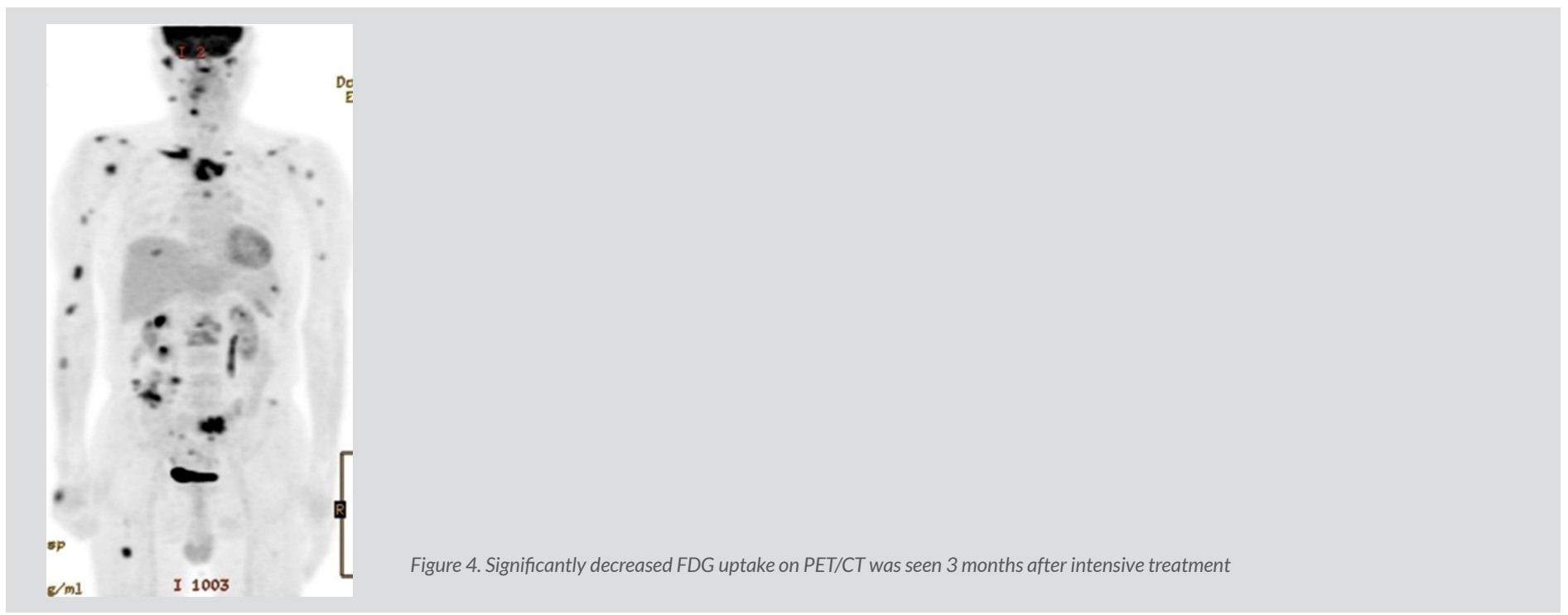

\section{DISCUSSION}

This patient had nasal symptoms 6 months before admission with a subsequent diagnosis of NTM infection based on biopsies of the right hand, left knee and nasal cavity during his hospitalisation. The patient presented with diverse manifestations, including hard palate perforation, multiple areas of eosinophilic infiltration in the iliac bone, granulomatosis and vasculitis in the nasal cavity, and foot drop with common peroneal neuropathy. However, these signs were not explained solely by a diagnosis of disseminated NTM infection. The patient was therefore diagnosed as having concomitant EGPA and NTM infection. PET/CT scans showed high FDG uptake in the nasal cavity, cervical lymph nodes and multiple areas in the bone/bone marrow with granulomatosis patterns, and significantly decreased FDG uptake with improved clinical manifestations after treatment (Fig. 3A, 3B).

Granulomatosis with polyangiitis (GPA), microscopic polyangiitis and EGPA are characterised by the presence of a positive ANCA and are therefore referred to as ANCA-associated vasculitis (AAV) ${ }^{[4,6,9]}$. Regardless of ANCA presence, a PET/CT scan in patients with AAV shows positive findings in multiple sites, including the lung, nasopharynx, joints, aorta, bone marrow and lymph nodes ${ }^{[6]}$. Although it has been reported that disseminated NTM infection may be associated with widespread FDG uptake in the lymph nodes and in multiple bony lesions ${ }^{[8]}$, FDG uptake in our patient was increased in the nasal cavity, a site which is commonly involved lesion related to AAV[3,4,12]. In addition, FDG uptake in our case revealed multiple spine lesions with skipped regions unlike disseminated NTM infection. These findings were presented on the FDG-PET/CT scan in our patient, and are not associated with disseminated NTM infection alone ${ }^{[10]}$.

Infections have been presumed to contribute to the development of some cases of vasculitis ${ }^{[10,11]}$. For example, hepatitis B virus, hepatitis $\mathrm{C}$ virus, Staphylococcus aureus and tuberculosis have all been associated with the onset and reactivation of vasculitis ${ }^{[11]}$. However, the mechanisms by which such infectious pathogens induce or exacerbate vasculitis are not fully understood ${ }^{[9-11]}$.

To the best of our knowledge, this is the first case report of a patient with EGPA combined with disseminated NTM infection with findings in multiple sites mimicking metastatic malignancy. In the future, more data are needed in a larger sample of patients to understand characteristic findings on FDG-PET/CT scans with respect to infection-induced vasculitis. Such information can better inform accurate diagnosis for the initiation of early and effective treatment.

\section{CONCLUSION}

We report a case of a patient with EGPA and disseminated NTM infection and describe the associated 18FDG-PET/CT scan features. 


\section{REFERENCES}

1. Lee SJ, Jeong JH, Lee CH, Ahn BC, Eun JS, Kim NR, et al. Development and validation of an (18) F-fluorodeoxyglucose-positron emission tomography with computed tomography-based tool for the evaluation of joint counts and disease activity in patients with rheumatoid arthritis. Arthritis Rheumatol 2019;71:1232-1240.

2. Ito K, Minamimoto R, Yamashita H, Yoshida S, Morooka M, Okasaki M, et al. Evaluation of Wegener's granulomatosis using 18F-fluorodeoxyglucose positron emission tomography/computed tomography. Ann Nucl Med 2013;27:209-216.

3. Kemna MJ, Vandergheynst F, Vöö S, Blocklet D, Nguyen T, Timmermans SA, et al. Positron emission tomography scanning in anti-neutrophil cytoplasmic antibodies-associated vasculitis. Medicine 2015;94:e747.

4. Soussan M, Abisror N, Abad S, Nunes H, Terrier B, Pop G, et al. FDG-PET/CT in patients with ANCA-associated vasculitis: case-series and literature review. Autoimmun Rev 2014;13:125-131.

5. Nelson DR, Johnson GB, Cartin-Ceba R, Specks U. Characterization of F-18 fluorodeoxyglucose PET/CT in granulomatosis with polyangiitis. Sarcoidosis Vasc Diffuse Lung Dis $2015 ; 32: 342-352$

6. Del Giudice G, Bianco A, Cennamo A, Santoro G, Bifulco M, Marzo C, et al. Lung and nodal involvement in nontuberculous mycobacterial disease: PET/CT role. Biomed Res Int 2015;2015:353202

7. Gioffredi A, Maritati F, Oliva E, Buzio C. Eosinophilic granulomatosis with polyangiitis: an overview. Front Immunol 2014;5:549.

8. Sohn S, Shi HJ, Wang SH, Lee SK, Park SY, Lee JS, et al. Mycobacterium avium complex infection-related immune reconstitution inflammatory syndrome mimicking lymphoma in an human immunodeficiency virus-infected patient. Infect Chemother 2018:50:350-356.

9. van Timmeren MM, Heeringa P, Kallenberg CG. Infectious triggers for vasculitis. Curr Opin Rheumatol 2014;26:416-423.

10. Lin K-H, Wang J-H, Peng N-J. Disseminated nontuberculous mycobacterial infection mimic metastases on PET/CT scan. Clin Nucl Med 2008;33:276-277. 